\title{
A Case of Primary Malignant Melanoma of the Palatine Tonsil M Milojević ${ }^{1}$, C Špadijer-Mirković ${ }^{1}$, A Perić ${ }^{1}$, B Vukomanović-Đurđević ${ }^{2}$, D Đerić ${ }^{3}$, J Trifunović ${ }^{4}$
}

\begin{abstract}
Primary malignant melanoma of the oropharyngeal mucosa is clinically very aggressive tumour caused by malignant transformation of melanocytes originating from the mucosal epithelium. We described a case of extremelly rare primary malignant melanoma of the palatine tonsil in a 71year-old man. The main symptoms were dysphagia and odynophagia. Diagnosis was done by pathohistological examination of the excised tonsil and supported by immunohistochemical analysis. Clinical examination didn't show another site with primary melanoma. To our knowledge, only five cases of primary tonsillar melanoma have been previously reported in the world literature. We discussed the clinical and histopathological characteristics of this tumour, as well as diagnostic and treatment options in the management of these rare lesions. Histopathological and immunohistochemical analysis and clinical data are necessary for diagnosis of primary oropharyngeal mucosal melanoma.
\end{abstract}

Keywords: Diagnosis; immunohistochemistry, malignant melanoma; primary; tonsil

From: ${ }^{1}$ Department of Otorhinolaryngology, Faculty of Medicine, Military Medical Academy, Belgrade, Serbia, ${ }^{2}$ Institute of Pathology, Faculty of Medicine, Military Medical Academy, Belgrade, Serbia, ${ }^{3}$ Department of Otorhinolaryngology and Maxillofacial Surgery, Clinical Centre of Serbia, Scool of Medicine, Belgrade, Serbia ${ }^{4}$ Clinical Hospital Centre "Zvezdara", Belgrade, Serbia.

Correspondence: Dr C Špadijer-Mirković, Department of Otorhinolaryngology, Faculty of Medicine Military Medical Academy, Crnotravska 17, 11040 Belgrade, Serbia. E-mail: cecaspadijermirkovic@gmail.com 


\section{INTRODUCTION}

Melanoma is tumour which mostly affects skin, but there is extracutaneous melanoma which includes ocular melanoma, melanoma arising from the mucosa of the digestive and nasal/paranasal sinuses mucosa, and leptomeningeal melanoma $(1,2)$. Primary mucosal melanoma is more frequent in women compared to men (1). Although there is no racial predilection, Afro-Americans and Asians appear to be proportionately more commonly affected than Caucasians (1). Main risk factor for primary oropharyngeal mucosal melanoma is smoking. It has been demonstrated that oral pigment leisons are more present among smokers (3). Primary oropharyngeal mucosal melanoma can affect many sites of oral cavity and oropharynx, but hard palate and maxillar gingiva are most commonly seen locations $(4,5)$. On the other hand, lips, toungue, palatine tonsils and uvula are extremelly rare primary sites of mucosal melanoma (5). To our knowledge, only five cases of primary malignant melanoma of the palatine tonsil have been reported in the literature $(6,7)$. Primary oropharyngeal mucosal melanoma usually presents as a dark brown or black lesion. However, $15 \%$ of these tumours are non-pigmental and typically red lesions, thus presenting as amelanotic melanomas that mimic some other oral mucosa red lesions (4).

\section{CASE REPORT}

A 71- year-old male Caucasian was admitted to the our ENT Department because of a rapidly enlarging lesion with ulceration arising from the right palatine tonsil with sensation of foreign body in the throat, dysphagia and light odynophagia. Earlier, he had also noticed a painless rightside cervical mass. Up until than he had always been a healthy person, except cigarette smoking and sensoneural hearing loss. On examination, a soft-tissue red leison, measuring up to $15 \mathrm{~mm}$ in 
diameter with ulceration of the mucosa was found on the medial surface of the right palatine tonsil. Soft and hard palate and tongue were apparently free of disease. By neck palpation, we found a jugular node on the right side, hard and painless to palpation, with dimension of approximatelly $1,5 \times 1,5 \mathrm{~cm}$. Computerized tomography (CT) scan showed a soft-tissue mass arising from the right palatine tonsil with dimension of $2 \times 1,6 \mathrm{~cm}$ and an upper jugular lymph node with dimension od $15 \times 13 \mathrm{~mm}$, and one node with suspect malignant characteristic in medial jugular group behind the sternocleidomastoid muscle with $14 \times 10 \mathrm{~mm}$ in diameter (Figure $1 \mathrm{a}, \mathrm{b}$ ).
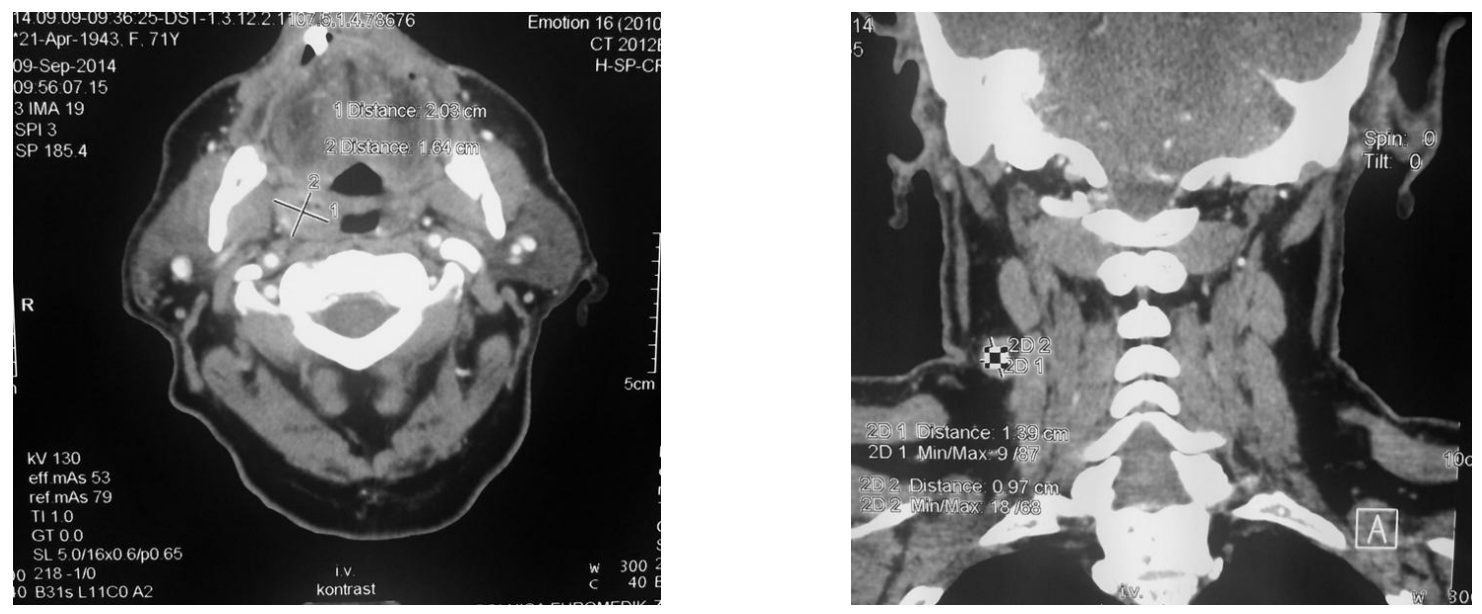

Fig.1: CT scan of the pharynx showed a soft-tissue mass arising from the right palatine tonsil with dimension of $2 \times 1,6 \mathrm{~cm}$ (a). CT scan of the neck demonstrated a upper jugular lymph node with dimension od $15 \times 13 \mathrm{~mm}$, and one node with suspect malignant characteristic in medial jugular group behind the sternocleidomastoid muscle (b).

Bilateral tonsillectomy under general anaesthesia was performed and it was uneventful. On operation, it was easy to separate tonsils from their bed. There was no major bleeding and macroscopically, after tonsillectomy, empty tonsillar beds seemed normal. The capsules seemed to be preserved. 
On histopathological examination, the right tonsil contained a solid malignant neoplasm of mixed epitheloid and spindle cell pattern. Microscopical image showed the presence of high grade tumour occupying the largest part of the superficial layer of tonsillar tissue. The tumour was growing from surface into the submucosa. There was an ulceration on the surface of the epithelial layer and, on the edge of ulceration, the epithelium was reactively hyperplastic. Tumour cells were anaplastic with discohesive solid growth. Pigment was not present (Figure 2).

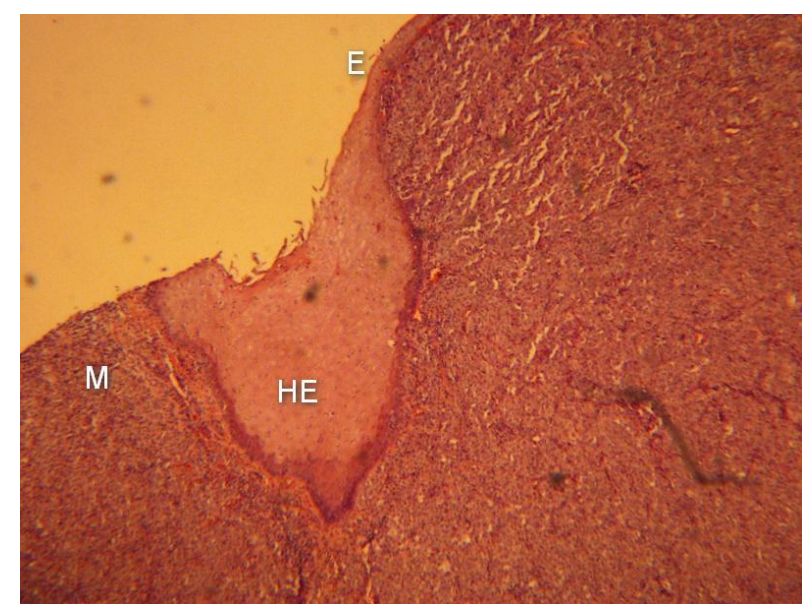

Fig.2: Histopathology showed the presence of high grade tumour occupying the largest part of the mucosal layer of tonsillar tissue. The tumour was growing from epithelium into the submucosa. There was an ulceration on the surface of the epithelial layer and, on the edge of ulceration, the epithelium was reactively hyperplastic. Tumour cells were anaplastic with discohesive solid growth. Abbreviations: Enormal epithelium; HE-hyperplastic epithelium; M-melanoma.

After that, an immunohisochemical analysis was performed. The tumour cells showed a negative immunoreactivity for pancytokeratin and high immunoreactivity for Melan-A (Figure 3), S-100 (Figure 4), vimentin and HMB-45. The clinical, radiological, histopathological and immunohistochemical features confirmed the diagnosis of an primary invasive amelanotic malignant melanoma. The histological finding of left palatine tonsil was normal. 


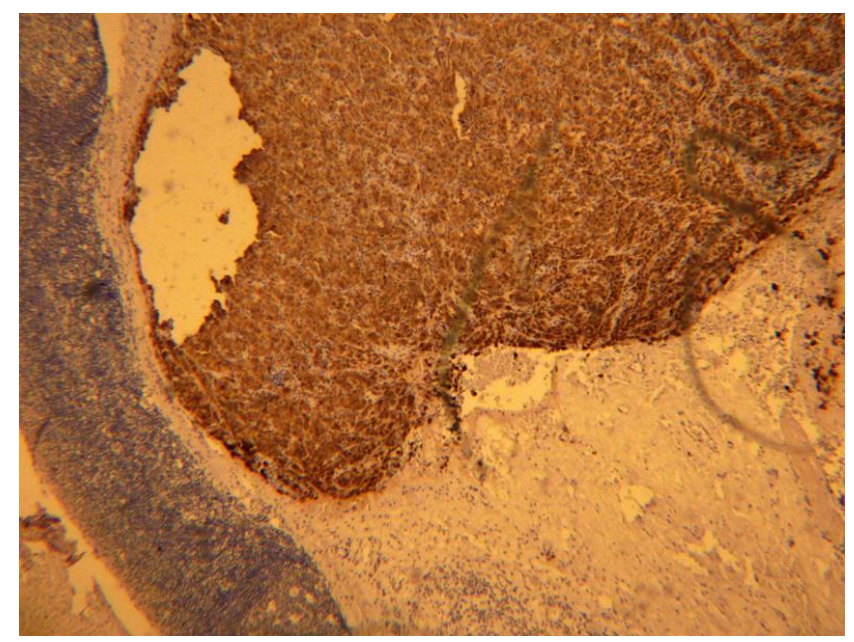

Fig.3: Immunohistochemical analysis showed the presence of amelanotic epitheloid tumour cells. The tumour cells showed high immunoreactitity for Melan A.

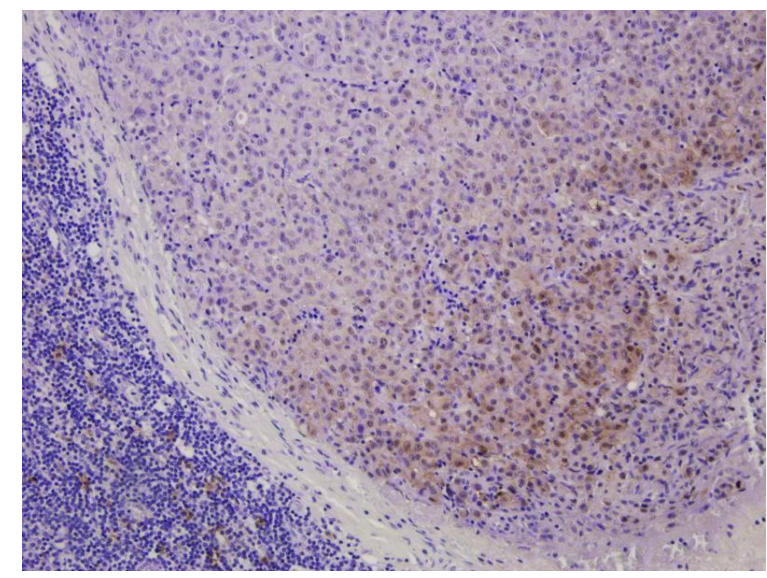

Fig. 4: The tumour cells were immunohistochemically positive for S-100.

The patient give no history of previously diagnosed cutaneous or mucosal melanomas at other sites. Further work-up, MRI studies of the chest and abdomen showed no evidence of distant metastasis or evidence of primary sites. After discussion with our oncological group, it was decided to perform neck dissection and to precede the treatment with local radiation and chemotherapy by tamoxifen $(20 \mathrm{mg} /$ day $)$. The patient refused any surgical treatment and agreed to radiation and chemotherapy. At the present time (seven months of follow-up) the patient is 
doing well and with no signs of local recurrence. The jugular lymph nodes are about the same size as before.

\section{DISCUSSION}

Mucosal melanomas are characterized by multiplicity, satelite formation, local and angiolymphatic invasion, consecutive pronounced local, regional and distant dissemination and a high rate of cause-specific death (5). Melanoma rarely becomes as a primary lesion of the oropharyngeal mucosa. It develops by uncontrolled proliferation of anaplastic atypical melanocytes situated in the basal layer of squamous epithelium. During embriologic development, melanocytes migrate from neural crest into the epitelium of the skin. As the oropharyngeal mucosa develops from an ectodermal depression or invagination, the epithelial lining of oropharyngeal mucosa, similar to the skin, contains melanocytes in its basal layer (8). However, it is noteworthy that nearly one third of mucosal melanomas in the head and neck region are amelanotic and are usually diagnosed in a locoregionally advanced clinical stage. At the time of presentation, the incidence of distant metastasis was reported to be $4-14 \%$ for mucosal melanomas and obout 5,3\% for skin melanomas (9). Accordingly, the prognosis of primary oropharyngeal melanomas is poor, with 5-year survival rates generally ranging from 10-25\% (10). There are numerous reasons for that poor prognosis: these neoplasms produce nonspecific symptoms, causing delay in the diagnosis; they affect old people, with inefficient immune systems; mucosae have rich lymphatic and vascular drainage, promoting early metastases; there are unusual anatomical constraints to the extent of surgery; these neoplasms seem to be histologically more aggressive, with a high mitotic index and marked anaplasia (5). The International Union Against Cancer (UICC) and the American Joint Committee on Cancer 
(AJCC) and World Health Organization (WHO) have not yet proposed clinical TNM classification for malignant melanomas and guidliness for oropharyngeal melanomas staging either (11). The Clark grading system assessing the depth of invasion and Breslow measuring the thickness of the tumour from the surface of the epidermis to the greathest depth of the tumour have no validation as prognostic factors in oropharyngeal melanomas due to rarity of these lesions (11). On the other hand, the traditional histologic staging for cutaneous melanoma can not be applied to the mucosal melanoma because of lacks histologic landmarks analogous to papillary and reticular dermis. Clinical stage, defined as localized tumours (Stage I, T any N0M0), tumours metastatic to regional lymph nodes (Stage II, $\mathrm{T}_{\text {any }} \mathrm{N} 1 \mathrm{M} 0$ ), and tumours metastatic to distant sites (Stage III, $\mathrm{T}$ any $\mathrm{N}$ any $\mathrm{M} 1$ ), appears to be the most significant prognostic factor (12). Prasad and co-authors proposed a clinical staging system with histopathological microstaging in three levels for stage I (with no regional lymph node metastases) (12). They defined the depth of invasion along three simple and reproducible categories: Level I: in situ mucosal melanoma without invasion or with microinvasion; Level II: invasion up to the lamina propria; and Level III: deep invasion into bone, cartilage, or skeletal muscle. The levels represent different microanatomic compartments separated by tissue barriers that are easily and reliably identifiable on light microscopy (e.g., Level I is predominantly above the epithelial basement membrane; Level II is below the epithelial basement membrane but above the perimysium, periosteum, or perichondrium; and Level III is invasion through the perimysium, periosteum, or perichondrium) (12). For all oral melanoma survival rate is $12.3 \%$ to $16.6 \%$ (13). Involvement of lymph nodes affects survival considerably with survival of 46 months, unless lymph nodes are not involved and 18 months when they are involved (13).

Differential diagnosis of primary oral melanoma includes melanotic maculae, oral naevy, smoking associated melanosis, amalgam tattoo, Addison's disease, Peutz - Jeghers syndrome and 
Kaposi's sarcoma $(5,7)$. On the other hand, it is important to differentiate primary from metastatic melanoma of the palatine tonsil. Metastases to the mucosal surfaces of the head and nech are rare. Tonsillar metastasis of melanoma malignum are also very rare and less than 30 cases was reported in the world literature (14). Metastatic melanoma may affect one or both tonsils in cases of disseminated melanoma. Primary and secondary melanomas of the mucosal surface of the upper aerodigestive tract may mimic each other and that careful microscopic examination is required to differentiate between them. The presence of an intraepithelial neoplastic component is main histological evidence supporting the clinical suspicion of a primary tumour. Microscopic examination of metastatic melanoma reveals an intact mucosal layer covering the tumour, while primary melanoma has junctional changes in the overlying mucosa (14). Therefore, ulceration and tumour progression may efface the junctional signs of primary melanoma. Before it may be concluded that a mucosal melanoma is a primary mucosal lesion, the presence of hidden, regressed, or previously removed cutaneous melanoma must be excluded (14). Our patient had no history of previously diagnosed cutaneous or mucosal melanomas at other sites.

Surgery is the main treatment option and it can be combined with radiotherapy and chemotherapy (15). The 5-year cause-specific survival probabilities increase (up to $48 \%$ ) on application of all the treatment modalities (9). Surgery is the primary treatment modality for localized mucosal melanomas, irrespective of the involement of the regional lymph nodes. Prophylatic neck dissection usually is not recommended, as mucosal melanomas of the head and neck region generally metastasize to the regional lymph nodes less frequently than do squamous carcinomas (9). The role of radiotherapy in the treatment of mucosal melanoma is not clearly defined. There has been a dogma that malignant melanoma is radioresistant tumour. However, 
Owens et al. (16) demonstrated that radiotherapy may have a beneficial effect in locoregional control of mucosal melanomas of head and neck region, although it does not provide the survival.

\section{CONCLUSION}

In conclusion, it has been described a case of primary mucosal malignant melanoma of the palatine tonsil. Considering poor prognosis and aggressive behavior of primary oropharyngeal malignant melanoma, it is necessary to make the diagnosis in early stage of disease. Good treatment results have been reported and it has been emphasized that the disease is potentially curable if diagnosed and treated at an early stage. Pathohistological and immunohistochemical findings and absence of cutaneous or mucosal melanomas at other sites are key for diagnosis of primary mucosal melanoma.

\section{Aknowledgement}

The authors declare no conflict of interest regarding the publication of this paper. 


\section{REFERENCES}

1. McLaughlin CC, Wu XC, Jemal A, Martin HJ, Roche LM, Chen VW. Incidence of noncutaneous melanomas in the U. S. Cancer 2005; 103: 1000-7.

2. Belli S, Yalcin S, Oktay MF. A case report: Primary melanoma of the nasal cavity. West Indian Med J 2013; 62: 778-80.

3. Axell T, Hedin CA. Epidemiologic study of excessive oral melanin pigmentation with special reference to the influence of tobacco habits. Scand J Dental Res 1982; 90: 434-42.

4. 4.Hicks MJ, Flaitz CM. Oral mucosal melanoma: epidemiology and pathobiology. Oral Oncol 2000; 36: 152-69.

5. Aguas SC, Quarracino MC, Lence AN, Lanfranchi-Tizeira HE. Primary melanoma of the oral cavity: ten cases and review of 177 cases from literature. Med Oral Patol Oral Cir Bucal 2009; 14: E265-271.

6. Xavier R, Paiva A, Ribeiro da Silva R, Gameiro dos Santos A. Primary malignant melanoma of the palatine tonsil: a case report. J Laryngol Otol 1996; 110: 163-6.

7. Das P, Kumar N, Ahuja A, Jain A, Ray R, Sakar C, Gupta SD. Primary malignant melanoma at unusual sites: an instututional experience with review of the literature. Melanoma Res 2010; 20: 233-9.

8. Auluck A, Zhang L, Desai R, Rosin MP. Primary malignant melanoma of maxillary gingiva - a case report and review of the literature. J Can Dent Med Assoc 2008; 74: 367 71.

9. Lemgyel E, Gilde K, Remenár É, Ésik O. Malignant mucosal melanoma of the head and neck - a review. Pathol Olcol Res 2003; 9: 7-12

10. Chidzonga MM, Mahomva L, Marimo C, Makunuke-Mutasa R. Primary malignant melanoma of the oral mucosa. J Oral Maxillofac Surg 2007; 65: 1117-20. 
11. Femiano F, Lanza A, Buonaiuto C, Gombos F, Di Spirito F, Cirilo N. Oral malignant melanoma: a review of the literature. J Oral Pathol Med 2008; 37: 383-8.

12. Prasad ML, Patel SG, Huvos AG, Shah JP, Busam KJ. Primary mucosal melanoma of the head and neck: a proposal for microstaiging localized stage I (lymph node-negative) tumors. Cancer 2004; 100: 1657-64.

13. Patrick RJ, Fenske NA, Messina JL. Primary mucosal melanoma. J Am Acad Dermatol 2007; 56: 828-34.

14. Barlas Aydogan L, Myers JN, Myers EN, Kirkwood J. Malignant melanoma metastatic to the tonsil. Laryngoscope 1996; 106: 313-6.

15. Van Lembergen EJ, Dok R, Laenen A, Hauben E, Van den Weyngaert D, Voordeckers M, De Neve W, et al. HPV-related oropharyngeal cancers in Flanders (Belgium): a multicenter study. B-ENT 2014; 10: 7-14.

16. Owens JM, Roberts DB, Myers JN. The role of postoperative adjuvant radiation therapy in the treatment of mucosal melanomas of the head and neck region. Arch Otolaryngol Head Neck Surg. 2003; 129: 864-8. 